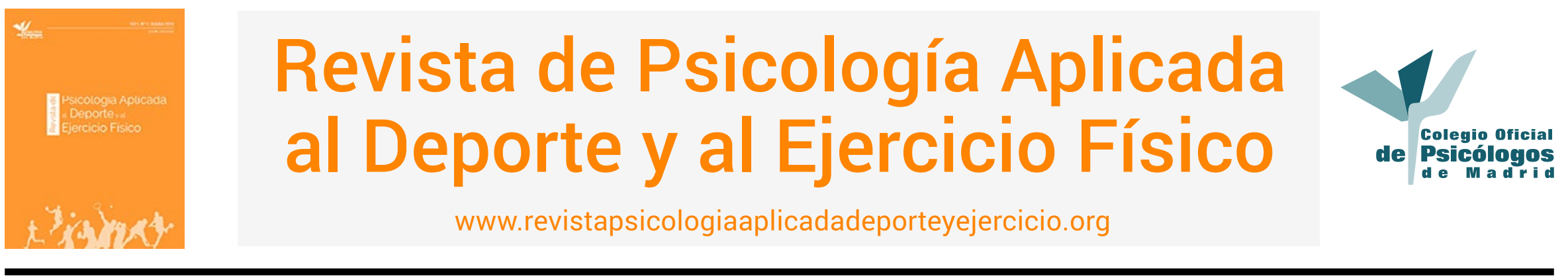

\title{
Entrenar a los padres, mejorar el equipo. La experiencia de la LIGA BRAVE
}

\author{
Juan González Hernández ${ }^{1,2}$, Pablo Martínez Mora² ${ }^{\text {, Martín Rodríguez }}{ }^{2}$ \\ 'Universidad de Granada, España, ${ }^{2}$ Liga Brave Vega Baja, España
}

RESUMEN: Reconocer cómo los esfuerzos que realizan los padres en cuanto a tiempo y dedicación en la práctica deportiva de sus hijos, resulta absolutamente relevante ante la organización del deporte infantil. Lo que durante décadas ha sido transmitido por la literatura científica, no parece tan evidente en el ámbito aplicado, donde la integración de la figura de los padres es muy escasa, incluso entendida como perjudicial en numerosas ocasiones. Desde hace ya 8 años, la LIGA BRAVE (Vega Baja del Río Segura, Alicante) persigue, a través de una competición en valores deportivos, que se desarrollen conductas deportivas en deportistas, padres y clubes de fútbol. Para ello, solicitamos la colaboración y participación de padres y clubes (cerca de 3000 personas y 44 clubes), para que, como modelos de comportamiento que son para los niños, atiendan al cumplimiento de normas conductuales que tendrán su repercusión para una clasificación final de Fair Play que se llevará paralelamente a la deportiva, y que supondrá la consecución de premios y reconocimientos a los jóvenes deportistas a final de temporada. A lo largo de todo el año, se van realizando diferentes propuestas de entrenamiento a los padres, que giran en torno a cinco objetivos específicos: a) generar un sentimiento positivo por la práctica del deporte; b) cómo mostrar el apoyo familiar a sus hijos; c) la utilidad que tienen los padres para favorecer el rendimiento deportivo; d) comprender la "doble carrera" rendimiento deportivo-escolar; y e) desarrollo de comportamientos positivos para convertirles en referencias en el deporte y la salud. Se concluye que los padres necesitan ser entrenados y asesorados como cualquier otra figura del ámbito del deporte infantil para mejorar la gestión de sus hijos, los comportamientos de deportividad y la comprensión de la labor de otros profesionales del ámbito, como el rol del entrenador y los árbitros.

PALABRAS CLAVES: Deporte de iniciación, padres, modelos de comportamiento y liga brave.

\section{Train the parents, improve the team. The experience of BRAVE LEAGUE}

ABSTRACT: Recognizition of how the efforts made by parents in terms of time and dedication to the practice of sport by their children is absolutely relevant for organization of children's sports. What the scientific literature has transmitted for decades does not seem so evident in the applied field, where integration of the figure of parents is very scarce, and even often understood as harmful. For eight years now, the BRAVE LEAGUE (Vega Baja of Segura River, Alicante) has pursued, through a competition in sports values, the development of sports behaviors in athletes, parents, and football clubs. For this purpose, we requested the collaboration and participation of parents and clubs (about 3000 people and 44 clubs), as behavioral models for children, by complying with behavioral norms. This will have an impact on a final classification of fair play scored in parallel to the sport activity. Based on the classification, young athletes will receive awards and recognitions at the end of the season. Throughout the year, parents are made different training proposals, which revolve around five specific objectives: a) to generate a positive feeling for the practice of sport; b) how to show family support to their children; c) the value of parents to favor sports performance; d) to understand the sports-school performance "double career"; and e) to develop positive behaviors to become references in sports and health. It is concluded that parents need to be trained and advised as any other

\footnotetext{
Financiación: Esta investigación fue financiada en parte con la ayuda del grupo de investigación "Psicología de la Salud/Medicina Conductual" (CTS-0267) concedida por la Consejería de Economía y Conocimiento de la Junta de Andalucía (España)

Juan González Hernández, es psicólogo. Universidad de Granada. Liga Brave Vega Baja; Pablo Martínez Mora, es entrenador de fútbol (nivel 3) y abogado. Liga Brave Vega Baja; Martín Rodríguez, es maestro de Educación Física. Liga Brave Vega Baja.

La correspondencia sobre este artículo debe enviarse al primer autor al Dpto. Personalidad, Evaluación y Tratamiento Psicológico. Grupo de Investigación Psicología de la Salud / Medicina conductual-CTS-0267. Facultad de Psicología. Campus de la Cartuja, s/n. 18071. Granada. España.
} 
figure in the field of children's sports to improve management of their children, sportsmanship behaviors, and understanding of the work of other professionals in the field, such as the role of the coach and referees.

KEYWORDS: Initiation sport, parents, behavior models, BRAVE LEAGUE.

\section{Treine os pais, melhore a equipa. A experiência da LIGA BRAVE}

RESUMO: Reconhecer os esforços dos pais, em termos de tempo e dedicação, relativamente ao desporto dos seus filhos é absolutamente relevante para a organização de desportos para crianças. Aquilo que durante décadas foi transmitido pela literatura científica, não parece ser tão evidente na aplicação em campo, onde a integração das figuras parentais é muito baixo, sendo mesmo entendida como prejudicial em muitos casos. Há 8 anos que a LIGA BRAVE (Vega Baja del Río Segura, Alicante) procura, através de uma competição em valores desportivos, desenvolver comportamentos desportivos em atletas, pais e clubes de futebol. Para tal, pedimos a colaboração e participação dos pais e clubes (cerca de 3000 pessoas e 44 clubes), para que, como modelos de comportamento que são para as crianças, demonstrem o cumprimento de normas de comportamento que terão o seu impacto para a classificação final de Fair Play que será atribuída em paralelo à classificação desportiva, implicando a conquista de prémios e reconhecimentos para os jovens atletas no final da temporada. Ao longo de todo o ano, diferentes propostas de formação são feitas aos pais, as quais giram em torno de cinco objetivos específicos: a) gerar um sentimento positivo para a prática de desporto; b) como mostrar o apoio familiar aos seus filhos; c) a utilidade que os pais têm para favorecer o desempenho desportivo; d) compreender o duplo percurso de desempenho desportivo e escolar; e e) desenvolvimento de comportamentos positivos para se tornarem referências no desporto e na saúde. Conclui-se que os pais precisam ser treinados e orientados como qualquer outra figura no domínio do desporto infantil, para melhorarem a gestão dos seus filhos, os comportamentos de desportivismo e a compreensão do trabalho de outros profissionais da área, tais como o papel do treinador e os árbitros.

PALAVRAS-CHAVE: Desporto de iniciação, pais, modelos de comportamento, liga brave.

Artículo recibido: 19/03/2018 | Artículo aceptado: 10/05/2018

El proceso de influencia social que un niño recibe cuando comienza sus primeros contactos con el deporte, contempla bien la denominada socialización deportiva, bien la socialización a través del deporte. El primer caso se refiere al proceso mediante el que la cultura deportiva es adquirida por los jóvenes hasta incorporar esta como parte de su personalidad (Cruz, Boixadós, Torregrosa y Mimbrero, 1996; Lorenzo, Cubero, López-Jiménez, y Hertting, 2017), dirigida a los principales agentes de socialización en el deporte (familia, educación formal, instituciones y entrenadores deportivos, grupo de iguales o medios de comunicación); el segundo alude al modo en que esa cultura deportiva, una vez adquirida, facilita al joven deportista una serie de mecanismos y recursos para integrarse de modo positivo en la sociedad (Bean, Fortier, Post, y Chima, 2014; Carbonero, Martín-Antón, Monsalvo, y Valdivieso, 2015; Vierimaa, Bruner, y Côté, 2018).

A modo de un "laboratorio de acción social" (con reglas, sus sanciones, éxitos, fracasos y demás situaciones que contribuyen a transferir esas cualidades y valores propios de la práctica deportiva), el Movimiento BRAVE supone una iniciativa en ambos caminos, surgiendo como un modelo diferente de competición deportiva, dejando de lado los resultados deportivos, y proponiendo la consolidación de conductas, razonamientos y actitudes prosociales en niños, padres y profesionales que trabajan para el deporte infantil, como un modelo de educación deportiva aplicado y posible.

El entorno familiar (padres en adelante) en el deporte ofrece influencias socializadoras sobre la formación de características personales del niño/a (Carreres, Escartí, Cortell, Fuster, y Andreu, 2012), habiéndose relacionado con la percepción de habilidad (Boixadós, Cruz, Torregrosa, y Valiente, 2004; Horn, 1985, Smith, Smoll y Curtis, 1979), respuestas emocionales y afectivas (Brustad, 1988; Partridge y Wann, 2015; Omli y La Voi, 2009; Scanlan y Lewthwaite, 1986), la participación, motivación y grado de implicación en el deporte o en la retirada del mismo (Cruz, 2001; Fraser-Thomas, Côté, y Deakin, 2008; Gimeno, 2007; Ortíz, 2017; Smoll, Smith, y Cumming, 2007), en la transmisión de valores y en el desarrollo moral (Brunelle, Danish, y Forneris, 2007; Turnnidge, Côté, y Hancock, 2014) o la percepción del éxito deportivo (Carratalà, Gutiérrez, Guzmán, y Pablos, 2011; Keegan, Spray, Harwood y Lavallee, 2010; Torregrosa et al., 2007). Tales referencias psicológicas, facilitarán la preparación que reciban los jóvenes no sólo como deportistas, sino también como personas, estableciéndose y generalizando preferencias sobre la funcionalidad de la personalidad de los jóvenes (Luciano, Gómez-Becerra, y Valdivia, 2002), en cuanto a sus procesos madurativos (ver Figura 1). 


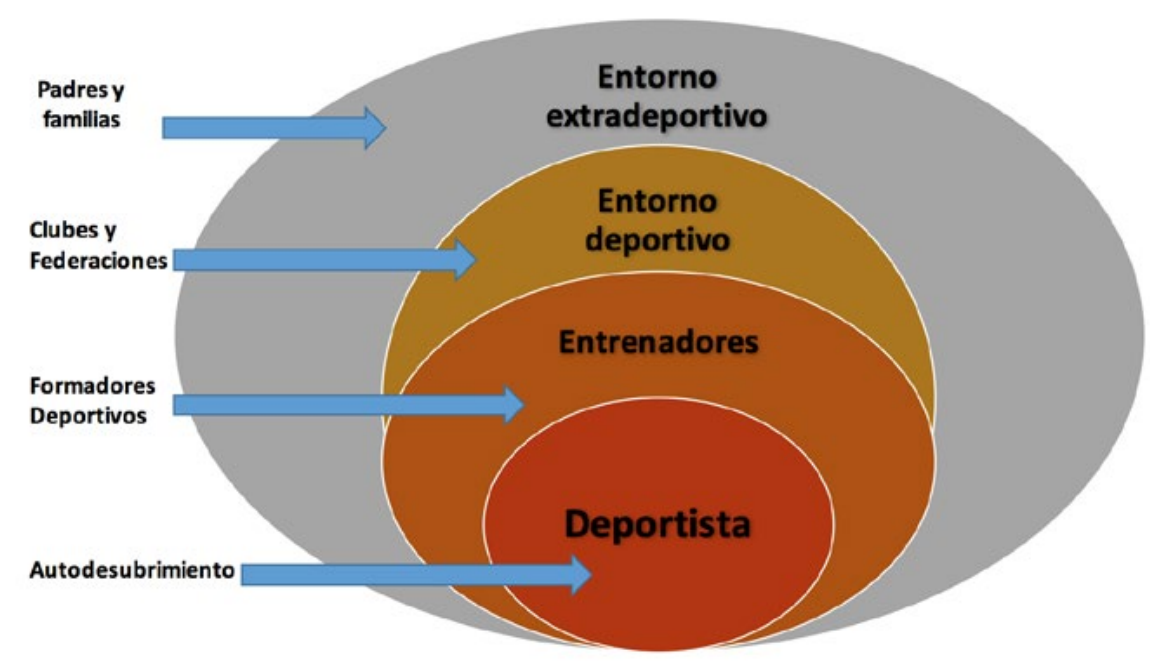

Figura 1. Influencias contextuales sobre la maduración del joven deportista (elaboración propia).

\section{Liga BRAVE, y sus procesos de integración de los padres como miembros del equipo deportivo}

La Liga BRAVE, con la colaboración de equipos de fútbol base de la Vega Baja del Río Segura (Alicante, España), y promovido por su Área Psicoeducativa, ha diseñado y puesto en marcha un modelo diferente de competición en valores depor- tivos y el señalamiento (a través de recompensas, reducciones de refuerzos, propuestas psicoeducativas de prevención e intervención), una estructura y organización competitiva de la práctica del fútbol base, donde se desarrollen conductas deportivas en deportistas, padres y clubes de fútbol. Para ello, la colaboración y participación de padres y clubes, que como modelos de comportamiento adulto para sus hijos atienden al cumplimiento de estas simples normas que tendrán su repercusión para la Clasificación Fair Play planteada paralelamente a la deportiva, y que supondrá la consecución de premios y trofeos a final de temporada.

El principal objetivo de las actuaciones psicoeducativas (Figura 2) es promover el juego limpio entre los participantes, creando un modelo de valores positivos y adecuados para la convivencia y el respeto a través del deporte, y utilizando el fútbol base como herramienta formativa para el desarrollo moral, educativo y psicológico.

La propuesta competitiva no pertenece a federación alguna, y se dirige a la práctica principalmente del fútbol y fútbol sala (Fútbol-6 para jugadores prebenjamines y Fútbol-8 para las categorías benjamín, Alevín e Infantil), aunque a lo largo de sus 7 años de existencia se ha abierto a la práctica de otras modalidades deportivas (baloncesto, balonmano, voleibol,...). En la

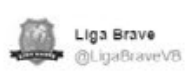

Muy pronto, haré con papá, mamá, entrenador y amigos, cosas que nos harán más grandes \#MovimientoBrave \#SonrisasBrave \#J9Brave (a) Asc SONRISAS

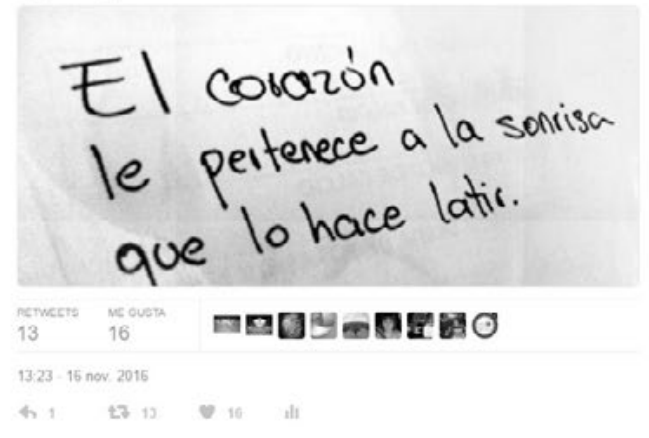

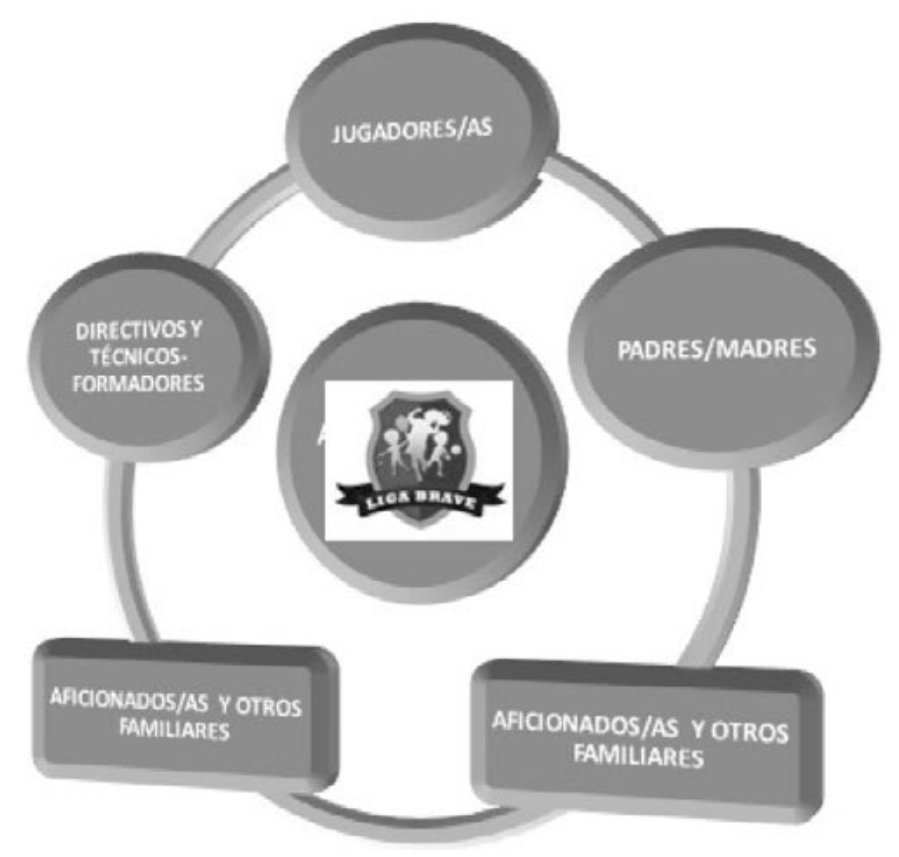

\section{Actuaciones}

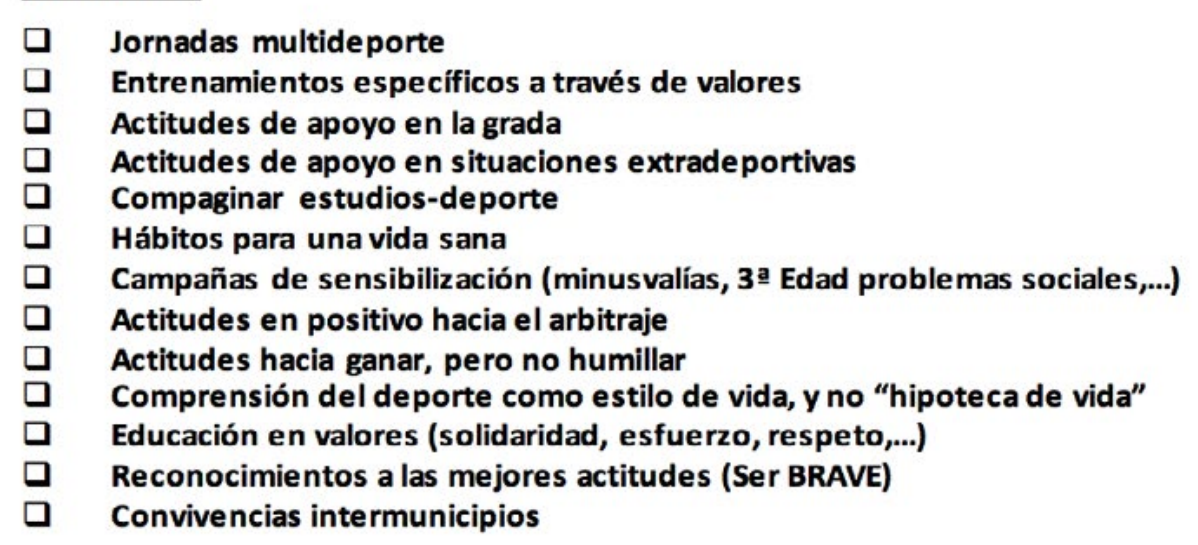

Figura 2. Actuaciones de la Liga BRAVE con todas las figuras del deporte base (elaboración propia). 
misma, participan todos aquellos niños y niñas pertenecientes a 27 municipios de la comarca (alrededor de 3000 niños/as), que no pertenecen a los clubs o equipos federados, y de esta manera pueden disfrutar de la oportunidad de practicar deporte (de no existir, supondría que esos niños no realizaran práctica deportiva organizada alguna. el respeto, la aceptación de cualquier circunstancia de juego y la deportividad al rendimiento deportivo, por medio de la educación y trasmisión de valores tanto humanos como deportivos en sus jóvenes deportistas.

Generar el interés de los adultos por una implicación útil y saludable psicosocialmente (Hoover-Dempsey y Sandler, 1995; Merino Orozco, Arraiz y Sabirón, 2017) para que desde bien pronto los niños aprendan a entender los contextos sociales (deportivos, familiares, escolares,...), a detectar las claves que les digan cómo otros funcionan, lo que les gusta o disgusta, o lo que perpetuamos en las interacciones cotidianas con los hijos, son objetivos principales en las iniciativas con padres de Liga BRAVE. Esta experiencias, ponen el punto de mira en los padres como fuentes del proceso socializador, en su actuación directa e indirecta, en sus comportamientos reforzadores o sancionadores, estilos de crianza, lazos comunicativos y de relación afectiva o verbal (Capano y Ubach, 2013; Jiménez-Iglesias y Moreno, 2015; Rodrigo et al., 2004), incluso en las primeras preferencias de sus hijos en cuanto a gustos y deseos, de los que la práctica del deporte no será menos (Arthur-Banning, Wells, Baker, y Hegreness, 2009).

Los padres son principales factores de protección, permitiendo a sus hijos una "elección limpia" del deporte que deseen practicar, siendo modelos a seguir en el comportamiento infantil y adulto, apoyando y fomentando actitudes saludables en cuanto a la práctica deportiva, construyendo recursos funcionales hacia la dificultad, entre otros (Arthur-Banning et al., 2009; Cremades, Donlon, y Poczwardowski, 2013, González y Otero, 2005; Ortíz, 2017). Pero también lo son de riesgo (ante conductas disruptivas, percepciones narcisistas del aprendizaje o modelos de presión, entre otros) (Dorsch, Smith y Dotterer, 2016; Raimundi, Molina, de Figueroa, y Schmidt, 2017; Vella, Cliff, y Okely, 2014).

En la misma Liga BRAVE, se trabaja con las diferentes tipologías de padres (implicados, sobreimplicados, ausentes, "entrenadores en la banda", etc.) en circunstancias de iniciación deportiva, y para cada encontrar las posibilidades de utilidad e intervención con cada una de ellas (Romero, Garrido y Zagalaz, 2009). El planteamiento de acciones donde se cuenta con la integración de los padres (Figura 3), comienza por mostrar al mundo del deporte base (profesionales del entrenamiento y la formación y clubes deportivos de base) los esfuerzos cada vez más necesarios para una formación a padres (comprender sus roles y oportunidades de aprovechamiento, limitaciones, transferencias positivas y negativas del mundo adulto al mundo infantil...).

Seguidamente, desarrollar iniciativas y acciones que permitan la comprensión de utilidad de todas las posibles tipologías de padres (Romero et al., 2009), que son los responsables de la decisión final de que sus hijos hagan deporte y que ninguno desea el malestar para sus hijos, desde (p. ej., probablemente, los padres más desinteresados no aporten nada, aunque si deberían conocer cuánto de ese desinterés o interés esporádico no ayuda para la implicación de sus hijos hacia la importancia del deporte).

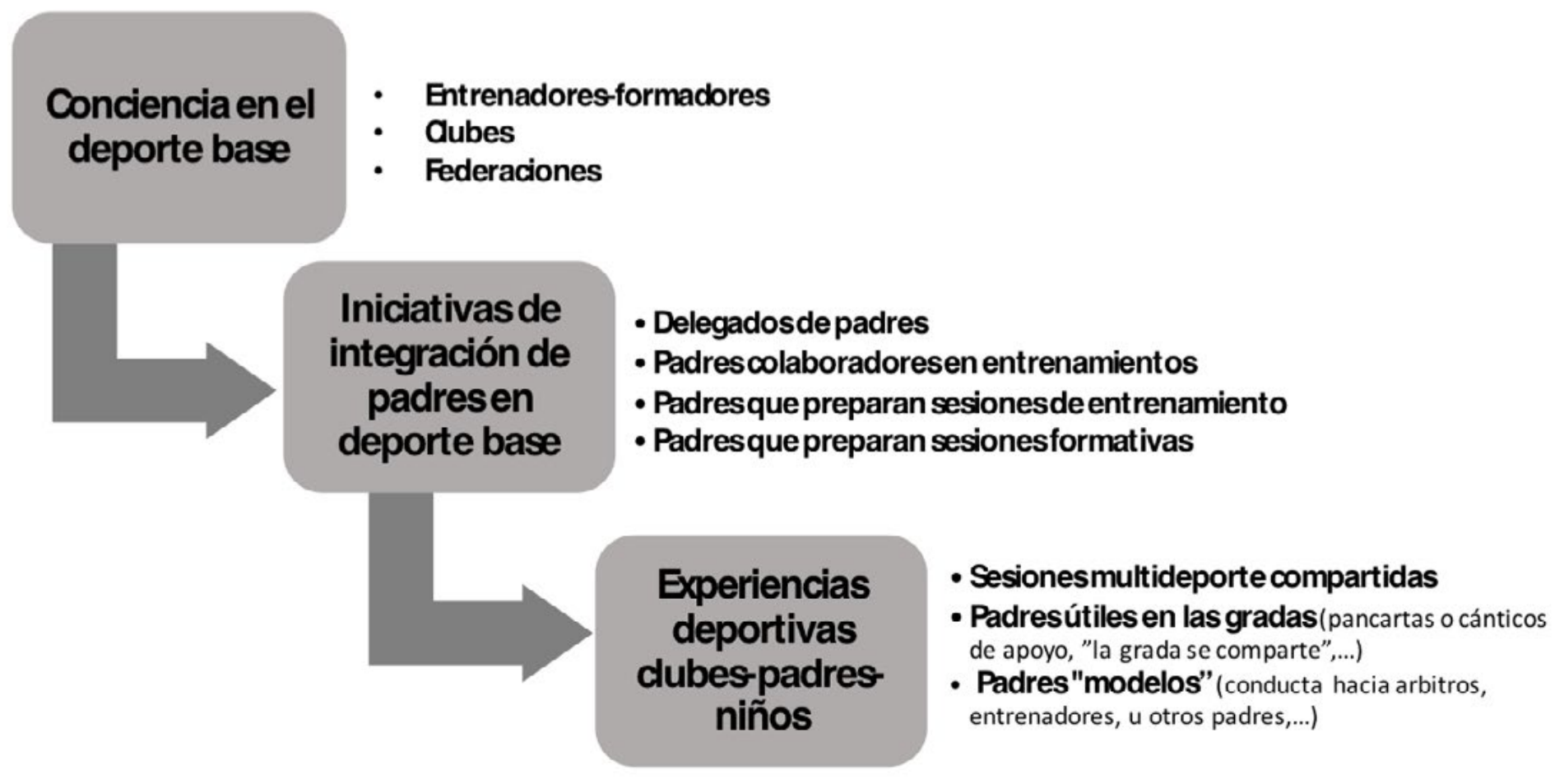

Figura 3. Procesos de integración de la figura de los padres en el deporte base (elaboración propia). 
Estudios que comparan familia, pares e influencia escolar o deportiva sugieren que la familia es más influyente que los pares en la escuela o el deporte (Teques y Serpa, 2009) mostrando relaciones convergentes entre percepciones de los hijos, cantidad de vinculación o interés de los padres hacia el deporte y los niveles de participación deportiva de los hijos (Marcos, Miguel, Oliva, Alonso, y Calvo, 2009; Ntoumanis, Taylor, y Thøgersen-Ntoumani, 2012). Por ello, crear el interés hacia la implicación en el deporte de alguno de los padres parece ser particularmente importante para los niños de su mismo género (Esteve, Musitu y Lila, 2005; Revuelta y Esnaola, 2011), de cara al nivel de importancia hacia el ejercicio físico o práctica deportiva de los jóvenes, o de la interpretación de su propia competencia y expectativas para de logro (Arthur-Banning et al., 2009; Harwood, y Knight, 2015; Teques y Serpa, 2009).

En la Liga BRAVE, se diseñan sesiones de trabajo con padres (Figura 4), donde se trabajan aspectos tan concretos como las diferentes tipologías de padres. Las citadas sesiones forman parte de actuaciones más globales, donde también se combinan sesiones con entrenadores y deportistas y responsables de clubes, Todas se complementan entre sí, al mismo tiempo que dan valor entre cada una de ellas.
Si ya de por sí, a medida que avanza la adolescencia, se produce un deterioro del estilo de vida saludable y un empeoramiento de las relaciones con la familia y con la escuela por parte del adolescente (Gutiérrez-Díaz del Campo, García-López, Chaparro-Jilete y Fernández-Sánchez, 2014), en el caso de las relaciones entre padres y de jóvenes deportistas, aparecen señales de implicación que únicamente aparecen ante tareas más cotidianas (como el rendimiento académico y deportivo), con expectativas poco ajustadas o realistas, depositan en sus hijos las principales fuentes de aparición de creencias perfeccionistas disfuncionales (Cremades et al., 2013, Rasquinha, Dunn, y Dunn, 2014), impulsividad por el logro de resultados (Carbonneau, Vitaro, Brendgen, y Tremblay, 2017; Smorti, y Guarnieri, 2014) o actitudes hacia el abandono deportivo (Amado, Sánchez-Oliva, González-Ponce, Pulido-González, y Sánchez-Miguel, 2015, Vella et al., 2014). De hecho, diversos estudios han revelado información importante acerca de las influencias positivas y negativas que ejercen sobre sus hijos/as, señalando expresiones de mayor disfrute cuando perciben que sus padres/ madres permiten el crecimiento personal (Holt, Tamminen, Black, Mandigo, y Fox, 2009; McCarthy y Jones, 2007) o de elevadas de ansiedad cuando los padres se sobreimplican

Ficha de Sesión de trabajo Escuela de Padres.

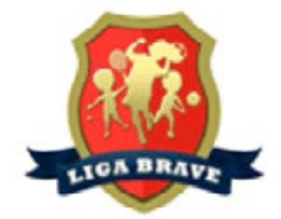

\section{TEMATICA}

Los padres que ante el cada vez más precoz comienzo de la relación de los niños con los contextos deportivos (cerca de los 4 años), serán los principales que acompañen a sus hijos en la construcción de hábitos psicosociales (actitudes y conductas) permanentes, la responsabilidad de la integración, y las posibilidad del mantenimiento a lo largo su trayectoria deportiva en la adolescencia, y la reducción significativa del abandono total de la práctica deportiva.

\begin{tabular}{l|l}
\multicolumn{1}{c}{ Tipología de padres } & Razones y mejoras \\
\hline Padres que han sido deportistas & \\
de élite & \\
\hline Padres interesados en la \\
formación deportiva como salud \\
\\
\end{tabular}

Figura 4. Sesión Escuela de Padres "Tipos de padres" (Romero et al., 2009). 
en las situaciones deportivas (Knight, Berrow, y Harwood, 2017; Shen et al., 2016).

\section{Utilidad para la implicación de los padres en el deporte de sus hijos}

Si bien es cierto que cuando existe implicación existen intereses (Wheeler, y Green, 2014), los padres centran todos sus esfuerzos en el bienestar de sus hijos, en este caso, en el contexto deportivo (Bean et al., 2014). En este sentido, se toman en cuenta dimensiones básicas como: a) exigencia paterna (control fuerte/control relajado), b) disposición paterna a la respuesta (reciprocidad, afecto/no reciprocidad, no afecto), o c) respuesta socio-emocional asociada (miedos / fortalezas, empatía / incomprensión).

A partir de estas premisas, el contexto deportivo del deporte base, necesita configurar las señales adecuadas en su organización y estructura, que aporten la relevancia y necesidad no de la práctica de un deporte únicamente porque sí (porque lo practicó su padre, madre o algún familiar relevante, porque es el más conocido, porque es el que más se practica en su lugar de residencia, etc.), sino de la constructiva comprensión para el joven deportista de lo que el deporte le reporta, principalmente a través de la diversión, satisfacción o sentido de utilidad.
Por ello, la principal responsabilidad del Área Psicoeducativa (equipo de psicólogos del deporte que trabajan de manera interdisciplinar con entrenadores) de Liga BRAVE, se centra en llevar a cabo un proceso constante de mediación entre todas las figuras del deporte base: coherencias o incoherencias entre quiénes estructuran el deporte (clubes y federaciones), quiénes entrenan (entrenadores-formadores) y quiénes asesoran (padres), paralelamente al trabajo directo que se realiza con los jóvenes deportistas. El entrenamiento de habilidades y recursos psicológicos y sociales es constante, facilitando la funcionalidad de los aprendizajes y mensajes exteriores que llegan al deportista (Mancini, Rigoli, Roberts, Heritage y Piek, 2017). A través de las intervenciones, se potencian procesos de regulación e influencia verbal y estrategias para la transferencia (descubrimiento guiado, p. ejemplo), el aprendizaje autónomo de cierta autorregulación verbal (autodiálogo), actitudes basadas en el aprendizaje de valoraciones sobre aspectos básicos de la interacción contextual y social (reconocimiento a través de las relaciones con otros) y de fortaleza individual (percepción de competencia) (Tabla 1).

Tales aspectos bien orientados, permitirán en los niños espacios donde puedan construirse a sí mismos, reconociendo sus limitaciones, apoyándose ante sus dificultades y trabajando hacia la mejora constante y autosatisfacción.

\section{Tabla 1. Principios para orientar hacia la utilidad del aprendizaje}

\begin{tabular}{|c|c|c|}
\hline Principios de utilidad en el aprendizaje & Conocimiento que se adquiere & Estrategias a seguir \\
\hline \multirow[t]{2}{*}{ RECIPROCIDAD Y COMPROMISO } & \multirow{2}{*}{$\begin{array}{l}\text { Construir la autopercepción que cada tarea } \\
0 \text { aprendizaje adquirido, forma parte de lo } \\
\text { que el joven deportista aportará a su madu- } \\
\text { ración, a sus mejoras personales y sociales, } \\
\text { así como una manera de aportarle recípro- } \\
\text { camente mejores y mayores recursos al } \\
\text { contexto (y los integrantes del mismo) que } \\
\text { le han permitido tales aprendizajes. }\end{array}$} & $\begin{array}{l}\text {-Crear sentimientos y creencias basadas } \\
\text { en el grado de diversión que obtiene por la } \\
\text { participación deportiva, inversiones perso- } \\
\text { nales basadas en la confluencia social de } \\
\text { las experiencias con los iguales y comparti- } \\
\text { das con sus padres. }\end{array}$ \\
\hline & & $\begin{array}{l}\text {-Orientar hacia las oportunidades que } \\
\text { surgen al asumir los motivos iniciales, } \\
\text { aprender y trabajar por los beneficios de la } \\
\text { relación social que se establece. }\end{array}$ \\
\hline \multirow[t]{2}{*}{ ESFUERZO E IMPLICACION } & \multirow{2}{*}{$\begin{array}{l}\text { Basar actitudes sobre aspectos en los que } \\
\text { hay que desarrollar un esfuerzo orientado } \\
\text { hacia el margen de mejoras. Las actitudes } \\
\text { de esfuerzo se configuran para alcanzar } \\
\text { dificultades, para superar retos que se ob- } \\
\text { servan de otros o que uno mismo configura } \\
\text { sobre lo que se valora útil y posible. }\end{array}$} & $\begin{array}{l}\text {-Trabajar a través de retos que fomenten } \\
\text { la posibilidad, fomentar autopercepciones } \\
\text { que permitan la confianza en lo aprendido, } \\
\text { en lo consolidado, en lo practicado. }\end{array}$ \\
\hline & & $\begin{array}{l}\text {-Consolidar recursos de la utilidad en las } \\
\text { acciones que se realizan, y la coherencia } \\
\text { del esfuerzo para la comprensión de la } \\
\text { implicación. }\end{array}$ \\
\hline
\end{tabular}




\section{Escuelas de Padres. Aportaciones en el entrenamiento visible e invisible de sus hijos}

Todo lo expuesto con anterioridad, persigue la organización y composición de Escuelas de Padres donde poder describirse y trabajar directa e indirectamente en el entrenamiento y asesoramiento de recursos a aportar a sus hijos deportistas. Convertidos en el principal enlace entre aprendizajes y enseñanzas, los padres establecen las estructuras socio-cognitivas más cercanas para la construcción psicosocial, creencias de posibilidad y la maduración de sus hijos de cara a comprender el deporte y diferentes actitudes en los deportistas (Arthur-Banning et al., 2009; Ortíz, 2017; Wang, Chow, y Amemiya, 2017). La organización de las Escuelas de Padres supondrá, aparte de la oportunidad perfecta para tal instrucción, un espacio para la puesta en práctica y realidad de creencias, recursos y habilidades para el apoyo en los momentos deportivos de sus hijos deportistas, que trasladarán al resto de facetas de sus vidas (Turman, 2007; Wuerth, Lee, y Alfermann, 2004).

Las características principales para trabajar en las Escuelas de Padres, se centrarán en: a) cómo llegar a ellos, b) cómo ofrecerles los suficientes recursos de utilidad, c) la secuencia de sesiones y temáticas, así como d) la combinación de diferentes momentos y espacios a utilizar en el programación del entrenamiento a padres. Por ello, las actuaciones con los padres de la Liga BRAVE se llevan desde el aula (sesiones formativas) a la grada ("gradas ejemplares"), desde la grada hasta el campo ("arbitra tu esfuerzo"), desde el campo hasta casa ("ordena tu ocio"), en las que involucran a sus hijos, sirviéndoles como modelos de comportamiento adulto.

Es por ello, que a modo de recomendaciones, se trabajan con los padres estrategias y competencias que puedan ayudar a construir en sus hijos deportistas lo que se denominan Mentalidades del joven BRAVE (conjuntos de recursos psicológicos funcionales, basados en sus niveles de maduración a través de las experiencias deportivas, y alejadas de los tan poco útiles resultados deportivos) (Figuras 5). Las Mentalidades del joven que hace deporte, se establecen y consensúan en un continuo madurativo (el cumplidor de normas, el seguidor de huellas y el explorador incansable), basado en aspectos generales de los niños como la edad, la relación con los padres, y la maduración psicosocial (niveles comprensivos, de relación o creencias de autoeficacia, posibilidad y autonomía) (Cecchini, Fernández-Rio y Méndez-Giménez, 2015).

Para cada tipología, se diseñan fichas de trabajo en la que se trabaja con los padres en varias sesiones (Figura 6). Tras describir la utilidad que cada tipología de padre aporta a la mentalidad, se les instruye en como estructurar aspectos cotidianos, que facilitan la construcción de actitudes y recursos para manejar funcionalmente las experiencias con sus hijos dentro y fuera del deporte. Los padres participan en el diseño de tales sesiones, proponiendo temáticas e iniciativas a poner en marcha para las siguientes sesiones, según lo aprendido en cada una de ellas. De esta esta manera, se sienten partícipes e implicados en las acciones formativas, así como surgen nuevas acciones a implantar en jornadas posteriores, durante los partidos de sus niños o en cualquier momento de interacción con ellos y sus entrenadores.

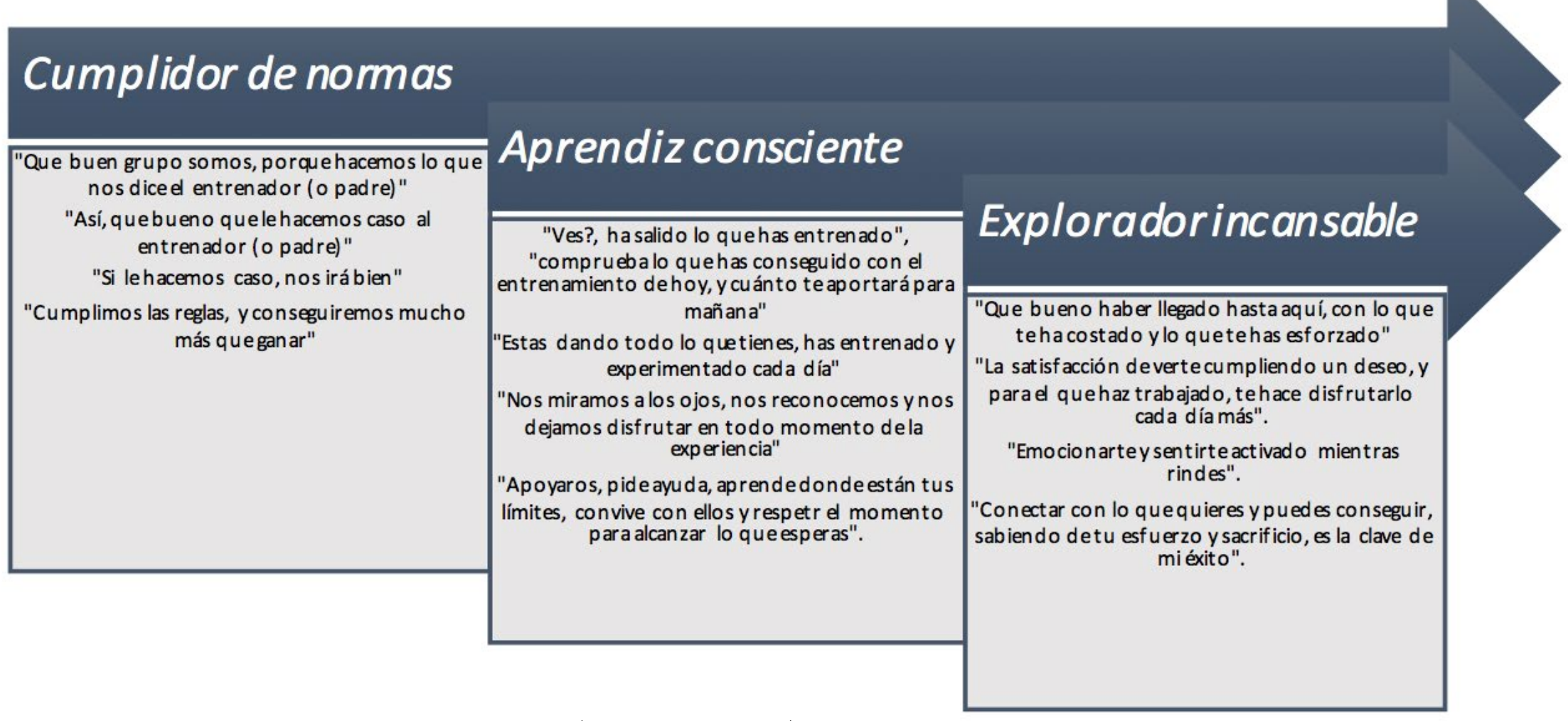

Figura 5. Mentalidades del joven deportista BRAVE (elaboración propia). 


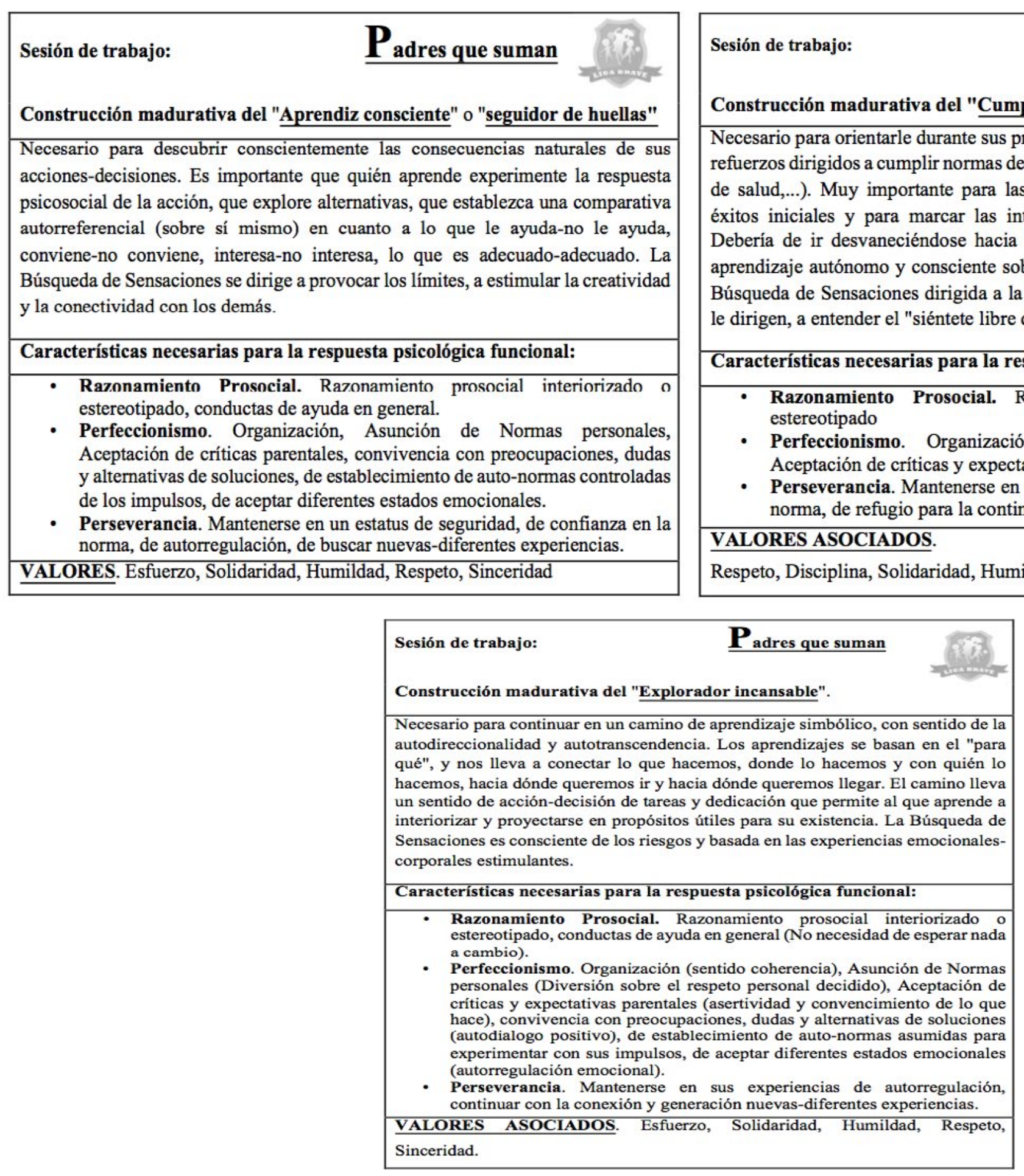

Figura 6. Mentalidades del joven que hace deporte. Sesiones Escuela de Padres (elaboración propia).

\section{La relevancia del Área Psicoeducativa en el desarrollo de Liga BRAVE}

El Área Psicoeducativa de Liga Brave, formada en la actualidad por 3 psicólogos (han llegado a formar parte hasta 5 psicólogos), que trabajan interdisciplinarmente con entrenadores y profesionales de los diferentes clubes, para la organización y puesta en marcha de las diferentes iniciativas que se generan. Los puntos fines principales de las funciones de esta son: a) la Educación y Formación dirigida a padres y jóvenes deportistas; b) la Planificación y Asesoramiento, donde las iniciativas tienen como objetivo a padres, entrenadores, y clubes; y c) la Evaluación Diagnostico e Interven- ción (cuando se considera necesario), para detectar y poner en marcha medidas ante situaciones concretas (bullying dentro de los equipos deportivos, actitudes hacia el dopaje, creencias sobre la valoración del éxito-fracaso en el deporte infantil, etc.), así como el seguimiento e interpretación de la clasificación Fair Play (clasificación de conductas y valores prosociales realizadas por niños, padres y entrenadores a lo largo de los partidos de toda una liga regular, valoradas por el árbitro y reflejadas en las actas de partido, y por la que se posicionan los equipos para la consideración final de resultado). En menor medida, se llevan a cabo algunos proyectos de investigación que, desde un punto de vista longitudinal, pretenden ir comprobando los cambios que se producen. 


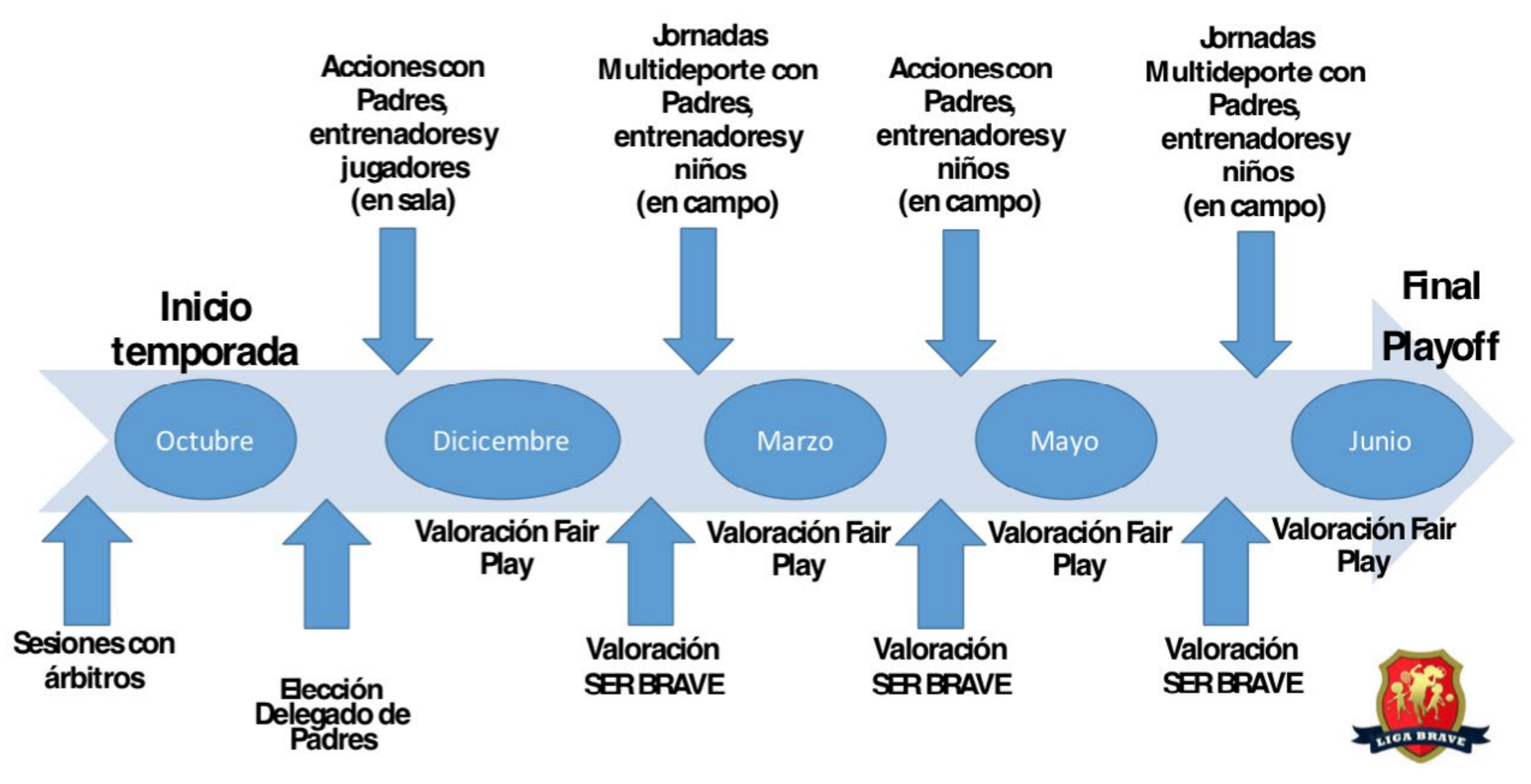

Figura 7. Cronograma de actividad Área Psicoeducativa Liga BRAVE (elaboración propia).

A la hora de desempeñar sus funciones, uno de los psicólogos es el responsable del área y dispone junto con el resto profesionales, la organización de actividades e idoneidad de las iniciativas. Al mismo tiempo, coordina el equipo de colaboradores a través de reuniones y sesiones donde se diseñan las actividades más adecuadas según criterios de planificación (Figura 7), o de prioridad a lo largo de tres trimestres. A lo largo de la temporada, se realizan diferentes iniciativas comunes (con todos los equipos), bajo el formato de Jornadas Multideporte donde los padres realizan tareas y juegos junto a sus hijos y entrenadores, compitiendo contra padres, hijos y entrenadores de otros clubes, potenciando la vivencia de las experiencias competitivas en su versión más lúdica.

\section{Discusión}

La mayoría de las situaciones tratadas en la literatura científica, que muestran la utilidad de los padres para aportar recursos (comportamentales, actitudinales, y emocionales) a sus hijos en situaciones deportivas, mantienen que ser padre controlador conlleva consecuencias negativas como falta de motivación, aburrimiento, comportamientos agresivos o falta de compromiso (Amado et al., 2015; Gimeno, Sáenz, Ariño, y Aznar, 2007; Soenens y Vansteenkiste, 2010).
Por el contrario, los padres que apoyan coherentemente a sus hijos en su práctica deportiva, facilitarán recursos como la autonomía, la comprensión de normas morales, la autoestima, la toma de decisiones seguras en sus hijos (Appelton y Duda, 2016; Arthur-Banning et al., 2009; Sánchez-Miguel, Pulido-González, Amado, Sánchez-Oliva, y Leo-Marcos, 2014; Steca, Bassi, Caprara, y Delle Fave, 2011).

Los contextos familiares son los que ejercen la mayor influencia en las primeras prioridades vitales, y sobre las preferencias de los niños en cuanto al comportamiento y gustos. Precisamente por ello, los padres que introducen a sus hijos en contextos deportivos (bien porque ellos también están inmersos, bien porque desean que sus hijos se desarrollen en los mismos), deben ser conscientes de sus responsabilidades en cuanto al interés de sus hijos en la práctica deportiva que han elegido para ellos.

Si de la misma manera, cualquier contexto de influencia en el joven deportista (extradeportivo o deportivo) marca señales en el mismo sentido del control, enfatizará en ellos la percepción de ser dirigidos, de poca identificación con las tareas y los aprendizajes (Mancini et al., 2017), así como de los desajustes psicológicos derivados de las mismas (miedo al fallo, ansiedad por agradar o corresponder, baja confianza en los recursos propios, pensamientos negativos...).

Sin embargo, la oportunidad que tienen los contextos familiares para el fomento de recursos facilitadores y funcio- 
nales como por ejemplo compartir el tiempo libre, fomentar la comunicación entre padres e hijos (Raimundi et al, 2017), genera mayor libertad, competencia y bienestar en actividades de aprendizaje (Steca et al., 2011), así como mayor autoestima, autoeficacia, estabilidad emocional y ajuste psicológico general (Fagan, Day, Lamb, y Cabrera, 2014; Omli y La Voi, 2009).

Basándonos en algunas propuestas aplicadas (Gimeno, 2006; Gómez-Espejo, Aroca, Robles-Palazón y Olmedilla, 2017; González-Hernández, Tomás-Macanás, Olivares, y Marín Irles, 2014; Vincent y Christensen, 2015), los padres también podrán formarse y ser entrenados para conseguir ser mucho más útiles en el asesoramiento y apoyo que ofrecen a sus hijos, y a conseguir que tal enseñanza sea complementaria a la que ofrecen los profesionales de los contextos deportivos, atendiendo a una primera fase de sensibilización y toma de consciencia; una segunda donde se marcan objetivos, decisiones y soluciones de problemas; y a una tercera fase de ensayo y utilización de pautas de conducta a aplicar en contextos de casa o incluso en los deportivos (padres realizando deporte junto a sus hijos). Hacer que las familias participen mantiene una dualidad a prestar atención: cuanto más apoyan y más activamente se implican (participación positiva) más comportamientos directivos emiten (parte negativa), ante la presión y la eventual contradicción a los técnicos (Ortiz-Marholz, Chirosa, Martín, Reigal y García-Mas, 2016).

Mientras la participación positiva a través del apoyo contribuye un mayor compromiso en los jóvenes deportistas, la participación negativa a partir de los comportamientos directivos construye una percepción de coacciones sociales, miedo al fallo y a la crítica, la neutralización de recursos hacia la actitud de esfuerzo, o la aparición de pensamiento que potencian la intención de abandono de la actividad. La influencia de la percepción de comportamientos por parte de las familias en el compromiso deportivo de los deportistas, se ve relacionada con comportamientos de apoyo y compresión, que favorecen.

Iniciativas como las Escuelas de Padres o Entrenamientos a Padres, se han generalizado en la atención en las estructuras del deporte base. En dichos programas, el trabajo inter y multidisciplinar entre psicólogos, entrenadores, educadores y padres debe fomentar el entrenamiento de aspectos relacionados con las conductas que más influencia ejercen sobre sus hijos, así como de las consecuencias sobre actitudes (comportamientos), vivencias (presión o motivación), y decisiones sobre la exigencia de la práctica deportiva que realizan. Además, y sobre todo dirigido a los padres de los niños que se inician y continúan avanzando en sus progresiones futbolísticas, es un buen escenario para poner en práctica el manejo de situaciones reales, de cara a saber manejarlas siempre con positividad y capacidad de adaptación al entorno deportivo y personal, implicándose incluso en el desempeño de roles como el Delegado de Padres (asumiendo la labor de portavoz y representante ante el club o cuerpos técnicos) o Padre Referencia (como modelo de comportamiento a seguir o a consultar ante diferentes circunstancias deportivas entre padres y sus hijos deportistas).

Todo lo que no permita la construcción de contextos de equilibrio entre la enseñanza deportiva formal (por parte de entrenadores) y la informal (por parte de los padres o familiares), convertirá los contextos deportivos en contextos poco facilitadores y donde niños y adolescentes quedan expuestos a situaciones de estrés, confusas prácticas de disciplina (entre padres y entrenadores) o comprensión de sus propios recursos personales al servicio del aprendizaje deportivo. Por el contrario, la implicación y entendimiento de todas y cada una de las responsabilidades que generan los roles de los adultos, permitirá la maduración necesaria para una respuesta psicológica y conductual basada en recursos funcionales, favoreciendo así la adaptación más adecuada y duradera ante las exigencias (si las hubiere) que supongan las experiencias deportivas.

Todavía queda mucho por aportar desde el punto de vista científico y aplicado a la utilidad de los adultos al deporte infantil, donde el entrenamiento a todos los protagonistas es necesario. Donde la coherente confluencia entre los intereses, capacidades y responsabilidades de todos ellos, se antoja vital para hacer que la fase del deporte base cumpla con la utilidad social a la que está destinada (aprendizaje deportivo de la práctica de un deporte, consolidación del deseo intrínseco por el mantenimiento de hábitos saludables y de movimiento, preparación progresiva de capacidades para la respuesta comportamental y psicológica adecuada conforme se van alcanzando categorías y niveles de exigencia deportiva superiores- principalmente competitivas-, y donde los padres aportan una labor impagable).

Diseñar programas de actividades/dinámicas cortas en la que puedan participar niños, entrenadores y padres realizando, practicando actividades físicas a través de juegos de habilidad, de competitividad, de apoyo, darán oportunidad a que los niños puedan compartir experiencias deportivas junto a sus padres y entrenadores, viviendo momentos donde se difumina quien es el niño y el adulto mostrando a todos en el natural disfrute de la acción, y sin perder la competitividad como eje de cada juego. Transmitiendo así de primera mano, los valores que configuran el eje central de lo que permitirá construir una "mentalidad deportiva", con el que poder conectar a todos (padres, clubes y jugadores), e identificarlos con experiencias positivas en la práctica deportiva. 
Como se ha podido comprobar, una iniciativa como lo es Liga BRAVE, aporta gran cantidad de posibilidades de intervención psicológica y educación deportiva, aunque al mismo tiempo encuentra limitaciones en consonancia con aspectos de diversa índole. El Movimiento BRAVE, es un movimiento psicosocial donde la necesidad de apoyo económico lo hace y hará siempre más viable. Muchas de las iniciativas dependen de la existencia de patrocinadores de las mismas o del esfuerzo altruista y desinteresado de sus organizadores, aspectos que en su ausencia o imposibilidad, hacen que en ocasiones no pueda llevarse a cabo todo lo planeado (lamentablemente, esto ha ocurrido en diferentes momentos). Los ayuntamientos apoyan facilitando las infraestructuras (instalaciones, horarios y desplazamientos principalmente), y el resto de fondos se proveen de cuotas que los mismos clubes aportan (seguro de accidentes, tareas de gestión administrativas, de difusión y algunas acciones del Área Psicoeducativa). Precisamente este es uno de los principales retos de Liga BRAVE, la consolidación de la organización a través de la apuesta firme y decidida por patrocinios o mecenazgos externos.

Otra de las limitaciones encontradas inicialmente, que con el paso de los 8 años de existencia se ha convertido en una de las principales oportunidades de aprovechamiento de las iniciativas del Área Psicoeducativa es la cultura deportiva general en cuanto al deporte base (fútbol base en este caso). Tanto responsables de clubes, como entrenadores (en ocasiones son las mismas personas), como padres reciben la organización de una liga de estas características con la creencia de ser y tener las mismas reglas y condiciones que las federativas, y desde el primer día donde comienzan a ser informados y educados sobre una propia normatividad, sobre que su implicación en conductas adecuadas durante el juego (hacia los niños, hacia árbitros, hacia los rivales, etc.) sumarán puntos para la clasificación final Fair Play (multiplicando por tres cada punto además) de sus pequeños, clubes y municipios. Todo ello, hace que se encuentren más abiertos a la participación sana, ofreciéndose incluso a iniciativas propias (como lo fue crear un Delegado de Padres), así como ha permitido que entrenadores y responsables de clubes pidan el apoyo del entrenamiento y asesoramiento psicológico, como una parte más de sus estilos y modelos de entrenar y gestionar iniciativas propias de cada club, estableciéndose un trabajo muy interesante de cara a la mejora e instauración de habilidades para observar, transmitir, decidir por los niños, autorregular sus creencias, impulsos y emociones, así como reconocer la labor del psicólogo del deporte que junto a ellos, construye un verdadero movimiento lleno de elementos positivos para los más pequeños y sus familias.

\section{Referencias}

Amado, D., Sánchez-Oliva, D., González-Ponce, I., Pulido-González, J. J. y Sánchez- Miguel, P. A. (2015). Incidence of parental support and pressure on their children's motivational processes towards sport practice regarding gender. PloS One, 10, e0128015. https://doi.org/10.1371/journal.pone.0128015

Appleton, P. R. y Duda, J. L. (2016). Examining the interactive effects of coach-created empowering and disempowering climate dimensions on athletes' health and functioning. Psychology of Sport and Exercise, 26, 61-70. https://doi.org/10.1016/j. psychsport.2016.06.007

Arthur-Banning, S., Wells, M. S., Baker, B. L. y Hegreness, R. (2009). Parents behaving badly? The relationship between the sportsmanship behaviors of adults and athletes in youth basketball games. Journal of Sport Behavior, 32(1), 3-18. Recuperado de: https:// search. proquest.com/docview/215873472? accountid=14542

Baiocchi-Wagner, E. A. y Talley, A. E. (2013). The role of family communication in individual health attitudes and behaviors concerning diet and physical activity. Health Communication, 28, 193-205. https://doi.org/10.1080/10410236.2012.674911

Bean, C. N., Fortier, M., Post, C. y Chima, K. (2014). Understanding how organized youth sport may be harming individual players within the family unit: A literature review. International Journal of Environmental Research and Public Health, 11, 10226-10268. https://doi.org/10.3390/ijerph111010226

Boixadós, M., Cruz, J., Torregrosa, M. y Valiente, L. (2004). Relationships among motivational climate, satisfaction, perceived ability, and fair play attitudes in young soccer players. Journal of Applied Sport Psychology, 16, 301-317. https://doi. org/10.1080/10413200490517977

Brunelle, J., Danish, S. J. y Forneris, T. (2007). The impact of a sport-based life skill program on adolescent prosocial values. Applied Developmental Science, 17(1), 43-55. https://doi. org/10.1080/10888690709336722

Brustad, R. J. (1988). Affective outcomes in competitive youth sport: The influence of intrapersonal and socialization factors. Journal of Sport and Exercise Psychology, 10, 307-321. https:// doi.org/10.1123/jsep.10.3.307

Capano, Á. y Ubach, A. (2013). Estilos parentales, parentalidad positiva y formación de padres. Ciencias Psicológicas, 7(1), 83-95.

Carbonero, M. A., Martín-Antón, L. J., Monsalvo, E. y Valdivieso, J. A. (2015). School performance and personal attitudes and social responsibility in preadolescent students. Anales de Psicología, 31, 990-999. https://doi.org/10.6018/analesps.31.3.181161

Carbonneau, R., Vitaro, F., Brendgen, M. y Tremblay, R. E. (2017). The intergenerational association between parents' problem gambling and impulsivity-hyperactivity/inattention behaviors in children. Journal of Abnormal Child Psychology, 1, 1-13. https://doi.org/10.1007/s10802-017-0362-x

Carratalà, V., Gutiérrez, M., Guzmán, J. F. y Pablos, C. (2011). Percepción del entorno deportivo juvenil por deportistas, padres, entrenadores y gestores. Revista de Psicología del Deporte, 20, 337-352.

Carreres, F., Escartí, A., Cortell, J. M., Fuster, V. y Andreu, E. (2012). The relationship between out-of-school sport participation and positive youth development. Journal of Human Sport and Exercise, 7, 671-683. https://doi.org/10.4100/jhse.2012.73.07 
Cecchini, J. A., Fernández-Rio, J. y Méndez-Giménez, A. (2015). Connecting athletes' self-perceptions and metaperceptions of competence: a structural equation modeling approach. Journal of Human Kinetics, 46(1), 189-198. https://doi.org/10.1515/hukin-2015-0047

Cremades, J. G., Donlon, C. J. y Poczwardowski, A. (2013). Parental involvement and gender differences in the psychological profile of freshmen collegiate athletes. Journal of Sport and Health Science, 2(3), 160-167. https://doi.org/10.1016/j. jshs.2012.05.001

Cruz, J. (2001). Factores motivacionales en el deporte infantil y asesoramiento psicológico a entrenadores y padres. En Autor (Ed.), Psicología del deporte (pp. 147-176). Madrid, España: Síntesis.

Cruz, J., Boixadós, M., Torregrosa, M. y Mimbrero, J. (1996). ¿Existe un deporte educativo?: papel de las competiciones deportivas en el proceso de socialización del niño. Revista de Psicología del Deporte, 5, 111-134.

Dorsch, T. E., Smith, A. L. y Dotterer, A. M. (2016). Individual, relationship, and context factors associated with parent support and pressure in organized youth sport. Psychology of Sport and Exercise, 23, 132-141. https://doi.org/10.1016/j.psychsport.2015.12.003

Elliott, S. K. y Drummond, M. J. (2017). Parents in youth sport: what happens after the game? Sport, Education and Society, 22, 391-406. https://doi.org/10.1080/13573322.2015.1036233

Esteve, J. V., Musitu, G. y Lila, M. (2005). Autoconcepto físico y motivación deportiva en chicos y chicas adolescentes. La influencia de la familia y de los iguales. Escritos de Psicología, 7, 82-90.

Fagan, J., Day, R., Lamb, M. y Cabrera, N. (2014). Should researchers conceptualize differently the dimensions of parenting for fathers and mothers? Journal of Family Theory \& Review, 6, 390405. https://doi.org/10.1111/jftr. 12044

Fraser-Thomas, J., Côté, J. y Deakin, J. (2008). Understanding dropout and prolonged engagement in adolescent competitive sport. Psychology of Sport and Exercise, 9, 645-662. https://doi. org/10.1016/i.psychsport.2007.08.003

Gimeno, F. (2006). Asesoramiento y formación con entrenadores y padres de deportistas jóvenes. En J. Garcés, A. Olmedilla y P. Jara (coords.), Psicología y Deporte (pp. 603-618). Murcia, España: DM Libreto-Editor.

Gimeno, F. (2007). Descripción y evaluación preliminar de un programa de habilidades sociales y de solución de problemas con padres y entrenadores en el deporte infantil y juvenil. Revista de Psicología del Deporte, 12, 67-79.

Gimeno, F., Sáenz, A., Ariño, J. V. y Aznar, M. (2007). Deportividad y violencia en el fútbol base: un programa de evaluación y de prevención de partidos de riesgo. Revista de Psicología del Deporte, 16, 103-118.

Gómez-Espejo, V., Aroca, B., Robles-Palazón, F. J. y Olmedilla, A. (2017). Formación integral en la cantera del Real Murcia C.F.: Tutorías psicológicas. Revista de Psicología Aplicada al Deporte y al Ejercicio Físico, 2, E11. https://doi.org/ 10.5093/rpadef2017a 11

González-Suárez, M. y Otero, M. (2005). Actitudes de los padres ante la promoción de la actividad física y deportiva de las chicas en edad escolar. Cuadernos de Psicología del Deporte, 5, 173-195.
González-Hernández, J., Tomás-Macanás, R., Olivares, C. y Marín-Irles, F. (2014). Escuela Elche CF, educando en el deporte desde el primer paso. Comunicación en Cipaf 2014. Actas de II Congreso Internacional de Psicología Aplicada al Futbol y Deportes de Equipo. INEF Barcelona.

Gutiérrez Díaz del Campo, D., García López, L. M., Chaparro Jilete, R. y Fernández Sánchez, A. J. (2014). Aplicación del modelo de Educación Deportiva en segundo de Educación Primaria: percepciones del alumnado y el profesorado. Cuadernos de Psicología del Deporte, 14(2), 131-144.

Harwood, C. G. y Knight, C. J. (2015). Parenting in youth sport: A position paper on parenting expertise. Psychology of Sport and Exercise, 16, 24-35. https://doi.org/10.1016/j.psychsport.2014.03.001

Hirschhorn, D. K. y Loughead, T. O. (2000). Parental Impact on Youth Participation in Sport the Physical Educator's Role. Journal of Physical Education, Recreation \& Dance, 71(9), 26-29. https:// doi.org/10.1080/07303084.2000.10605718

Holt, N. L., Tamminen, K. A., Black, D. E., Mandigo, J. L. y Fox, K. R. (2009). Youth sport parenting styles and practices. Journal of Sport and Exercise Psychology, 37(1), 37-59.

Hoover-Dempsey, K. V. y Sandler, H. M. (1995). Parental involvement in children's education: Why does it make a difference? Teachers College Record, 97, 310-331.

Jiménez-Iglesias, A. y Moreno, C. (2015). La influencia de las diferencias entre el padre y la madre sobre el ajuste adolescente. Anales de Psicología, 37, 367-377. https://doi.org/10.6018/analesps.31.1.158081

Keegan, R., Spray, C., Harwood, C. y Lavallee, D. (2010). The motivational atmosphere in youth sport: Coach, parent, and peer influences on motivation in specializing sport participants. Journal of Applied Sport Psychology, 22(1), 87- 105. https://doi. org/10.1080/10413200903421267

Knight, C. J., Berrow, S. R. y Harwood, C. G. (2017). Parenting in sport. Current Opinion in Psychology, 16, 93-97. https://doi.org/10.1016/j.copsyc.2017.03.011

Lorenzo-Fernández, M., Cubero-Pérez, R., López-Jiménez, A. M. y Hertting, K. (2017). "Entrenando a familias". Revista de Psicología del Deporte, 27(3), 37-42.

Luciano Soriano, M. C., Gómez Becerra, I. y Valdivia Salas, S. (2002). Consideraciones acerca del desarrollo de la personalidad desde un marco funcional-contextual. International Journal of Psychology and Psychological Therapy, 2, 173-197.

Mancini, V., Rigoli, D., Roberts, L., Heritage, B. y Piek, J. (2017). The relationship between motor skills, perceived self competence, peer problems and internalizing problems in a community sample of children. Infant and Child Development, 1(1), 1-16. https://doi.org/10.1002/icd.2073

Marcos, F. M. L., Miguel, P. A. S., Oliva, D. S., Alonso, D. A. y Calvo, T. G. (2009). Interacción entre la percepción del comportamiento de los padres y los comportamientos deportivos en edades de iniciación. Accion Psicologica, 6(2), 55-62.

McCarthy, P. J. y Jones, M. V. (2007). A qualitative study of sport enjoyment in the sampling years. The Sport Psychologist, 21, 400-416. https://doi.org/10.1123/tsp.21.4.400

Merino Orozco, A., Arraiz, A. y Sabirón, F. (2017). La adherencia del entorno familiar en el fútbol prebenjamín: Un estudio de caso. Revista Iberoamericana de Psicología del Ejercicio y el Deporte, 12, 139-148. 
Ntoumanis, N., Taylor, I. M. y Thøgersen-Ntoumani, C. (2012). A longitudinal examination of coach and peer motivational climates in youth sport: Implications for moral attitudes, well-being, and behavioral investment. Developmental Psychology, 48, 213-223. https://doi.org/ 10.1037/a0024934

Omli, J. y La Voi, N. M. (2009). Background anger in youth sport: A perfect storm? Journal of Sport Behavior, 32, 242-260.

Ortíz, P. (2017). "No todo es Balón". Implicando positivamente a los padres en la práctica del fútbol en etapa formativa. Revista de Psicología Aplicada al Deporte y al Ejercicio Físico, 2, E9. https://doi.org/ 10.5093/rpadef2017a9

Ortiz Marholz, P. F., Chirosa Ríos, L. J., Tamayo, I. M., Enrique Reigal Garrido, R. y García Mas, A. (2016). Deporte extracurricular: examinando el compromiso en relación con variables socioeducativas. RETOS. Nuevas Tendencias en Educación Física, Deporte y Recreación, 29, 53-57.

Partridge, J. A. y Wann, D. L. (2015). Exploring the Shame Coping Experiences of Youth Sport Parents. Journal of Sport Behavior, 38(3). Recuperado de: https://www.thefreelibrary.com/Exploring+the+shame+coping+experiencestoftyouth+sport+parents.-a0426541909

Raimundi, M. J., Molina, M. F., de Figueroa, N. L. y Schmidt, V. (2017). La comunicación entre padres e hijos: su influencia sobre el disfrute y el flow adolescente. Revista de Psicología, 26(2), 1-14.

Rasquinha, A., Dunn, J. G. y Dunn, J. C. (2014). Relationships between perfectionistic strivings, perfectionistic concerns, and competitive sport level. Psychology of Sport and Exercise, 15, 659-667. https://doi.org/10.1016/j.psychsport.2014.07.008

Revuelta, L. y Esnaola, I. (2011). Clima familiar deportivo y autoconcepto físico en la adolescencia. European Journal of Education and Psychology, 4(1), 19-31. https://doi.org/10.30552/ejep. v4i7.61

Rodrigo, M. J., Máiquez, M. L., García, M., Mendoza, R., Rubio, A., Martínez, A. y Martín, J. C. (2004). Relaciones padres-hijos y estilos de vida en la adolescencia. Psicothema, 16, 203-210.

Romero, S., Garrido, M. E. y Zagalaz, M. L. (2009). El comportamiento de los padres en el deporte. Retos. Nuevas tendencias en Educación Física, Deporte y Recreación, 15, 29-34.

Sánchez-Miguel, P. A., Pulido-González, J. J., Amado, D., Sánchez-Oliva, D. y Leo-Marcos, F. M. (2014). Percepción de la conducta de los padres en los comportamientos antisociales mostrados por los jóvenes participantes de deportes colectivos. Universitas Psychologica, 13(1), 15-24. https://doi.org/ 10.11144/Javeriana.UPSY13- 1.pcpc

Scanlan, T. K. y Lewthwaithe, R. (1986). Social psychological aspects of competition for male youth sports participants: IV. Predictors of enjoyment. Journal of Sport Psychology, 8(1), 25-35. doi.org/10.1123/jsp.8.1.25

Shen, B., Centeio, E., Garn, A., Martin, J., Kulik, N., Somers, C. y McCaughtry, N. (2016). Parental social support, perceived competence and enjoyment in school physical activity. Journal of Sport and Health Science, 1(1), 1-7. https://doi.org/ 10.1016/j. jshs. 2016.01.003

Smith, R. E., Smoll, F. L. y Curtis, B. (1979). Coach effectiveness training: A cognitive-behavioral approach to enhancing relationship skills in youth sport coaches. Journal of Sport Psychology, 1(1), 59-75. https://doi.org/10.1123/jsp.1.7.59
Smoll, F. L., Smith, R. E. y Cumming, S. P. (2007). Effects of a motivational climate intervention for coaches on changes in young athletes' achievement goal orientations. Journal of Clinical Sport Psychology, 7(1), 23-46. https://doi.org/10.1723/jcsp. 1.7.23

Smorti, M. y Guarnieri, S. (2014). Sensation seeking, parental bond, and risky driving in adolescence: Some relationships, matter more to girls than boys. Safety Science, 70, 172-179. https://doi. org/10.1016/j.ssci.2014.05.019

Soenens, B. y Vansteenkiste, M. (2010). A theoretical upgrade of the concept of parental psycho- logical control: Proposing new insights on the basis of self-determination theory. Develop-mental Review, 30(1), 74-99. https://doi.org/ 10.1016/j. dr.2009.17.001

Steca, P., Bassi, M., Caprara, G. V. y Delle Fave, A. (2011). Parents' self-efficacy beliefs and their children's psychosocial adaptation during adolescence. Journal of Youth and Adolescence, 40, 320-331. https://doi.org/10.1007/s10964-010-9514-9

Teques, P y Serpa, S. (2009). Implicación parental: adaptación de un modelo teórico al deporte. Revista de Psicología del Deporte, 18, 235-252.

Torregrosa, M., Cruz, J., Sousa, C., Viladrich, C., Villamarín, F., Garcia-Mas, A. y Palou, P. (2007). La influencia de padres y madres en el compromiso deportivo de futbolistas jóvenes. Revista Latinoamericana de Psicología, 39, 227-237.

Turman, P. D. (2007). Parental sport involvement: Parental influence to encourage young athlete continued sport participation. Journal of Family Communication, 7(3), 151-175. https://doi. org/10.1080/15267430701221602

Turnnidge, J., Côté, J. y Hancock, D. J. (2014). Positive youth development from sport

to life: Explicit or implicit transfer?. Quest, 66, 203-217. https://doi. org/10.1080/00336297.2013.867275

Vella, S. A., Cliff, D. P. y Okely, A. D. (2014). Socio-ecological predictors of participation and dropout in organised sports during childhood. International Journal of Behavioral Nutrition and Physical Activity, 17(1), 1-10. https://doi.org/10.1186/1479-5868-11$\underline{62}$

Vierimaa, M., Bruner, M. W. y Côté, J. (2018). Positive youth development and observed athlete behavior in recreational sport. PLOS one, 13(1), 1-14. https://doi.org/10.1371/journal.pone.0191936

Vincent, A. P. y Christensen, D. A. (2015). Conversations with parents: A collaborative

sport psychology program for parents in youth sport. Journal of Sport Psychology in Action, 6(2), 73-85. https://doi.org/10.1080/ 21520704.2015.1054535

Wang, M. T., Chow, A. y Amemiya, J. (2017). Who wants to play? Sport motivation trajectories, sport participation, and the development of depressive symptoms. Journal of Youth and Adolescence, 46, 1982-1998. https://doi.org/10.1007/s10964-0170649-9

Wheeler, S. y Green, K. (2014). Parenting in relation to children's sports participation: generational changes and potential implications. Leisure Studies, 33, 267-284. https://doi.org/10.1080/0 2614367.2012 .707227

Wuerth, S., Lee, M. J. y Alfermann, D. (2004). Parental involvement and athletes' career in youth sport. Psychology of Sport and Exercise, 5(1), 21-33. https://doi.org/10.1016/S14690292(02)00047-X 